\title{
The Analysis of the Chinese Phrasal VR Constructions from the Perspective of Event Structure Theory
}

\author{
Lijuan Feng, Luyao Chen, Liping Feng \\ College of Chinese Language and Culture, Beijing Normal University, Beijing, China \\ Email: Ifeng@mail.bnu.edu.cn, harrychenlyzml@gmail.com, fengliping@bnu.edu.cn
}

Received 10 January 2015; accepted 10 February 2015; published 13 February 2015

Copyright (C) 2015 by authors and Scientific Research Publishing Inc.

This work is licensed under the Creative Commons Attribution International License (CC BY). http://creativecommons.org/licenses/by/4.0/

\section{(c) (i) Open Access}

\begin{abstract}
There is a kind of Chinese VR (verb-resultative) constructions in which the meanings of verb and complement (stands as the "result") are relatively independent and decomposable. We call it "phrasal VR construction". Syntactic structures of this kind are various. The semantic orientations of complements are complicated. And there are some grammatical phenomena that have not been explained yet. After choosing four typical Chinese VR structures (“哭累” (cry-tired, being tired from crying), “哭湿” (cry-wet, being wet from crying), “洗累” (wash-tired, being tired from washing), and “推倒” (push over)), this article first described them in accordance with Levin \& Rappaport's event structure theory, and summarized the principles of the three participants' prominence and recession. Then the article, by analyzing the projection process from the event structure to the syntactic structure, found the correspondence between the participants and the syntactic elements. Finally it gave interpretations to the unexplained grammatical phenomena.
\end{abstract}

\section{Keywords}

Phrasal VR Construction, Event Structure, Participant, Syntactic Structure, Projection

\section{Introduction}

There is a kind of Chinese VR (verb-resultative) constructions; the meanings of verb and complement (stands as the “result”) are relatively independent and decomposable. Taking “哭累” (cry tired, being tired from crying) for instance, “张三哭累了” (Zhang San cried tired; Zhang San was tired from crying) can be interpreted as “张三哭” (Zhang San cried) and “张三累了” (Zhang San was tired). We call this kind of construction “ phrasal VR constructions”, such as “看烦” (watch tired, being tired of watching), “压断” (crush broken, being broken from 
crushing), “玩忘” (play forgotten, being forgotten from playing), etc.

Syntactic structures of this kind of constructions are various, and the semantic orientations of complements are complicated. Thus, some grammatical phenomena have not been explained yet. For example, both (1) “哭累” and (2) “哭湿” are combined by the one-valence verb (which can only have one argument) “哭” and the onevalence adjective “累” or “湿”, but why can only the “哭湿” have an object? Another example is that both (3) “洗累” and (4) “推倒” are made up by the two-valence verb “洗” or “推” and the one-valence adjective “累” or “倒”; however, why can not “洗累” behave the same as “推倒”, taking an object directly, but have to repeat the main verb “洗” to bring the object forward? (“*” before the sentences stands for “ungrammatical”)

(1) 他哭累了。

He-cried-tired.

He was tired from crying.

*他哭累了他。

*He-cried-tired-him.

(2) 张三哭湿了手绢。

Zhang San-cried-wet-the handkerchief.

Zhang San cried the handkerchief wet.

*张三哭湿了。

*Zhang San cried wet.

(3) 妈妈洗衣服洗累了。

Mother-washed-the clothes-washed-tired.

Mother was tired from washing the clothes.

*妈妈洗累了衣服。

*Mother-washed-tired-the clothes.

(4) 有人推倒了椅子。

Someone-pushed-over-the chair.

Someone pushed over the chair.

*有人推椅子推倒了。

*Someone-pushed-the chair-pushed-over.

If we want to figure out the features of the phrasal VR constructions in a comparatively complete way, it entails to study these constructions at the deep semantic level, the event structure level. Actually, in terms of the event structure, the four VR constructions above represent four types of the Chinese phrasal VR constructions respectively, and we will analyze them concretely later on.

\section{Levin \& Rappaport's Event Structure Theory}

Event denotes the time and space-specific action, activity or change that can be perceived by the observers, including three types, activity, achievement and accomplishment, and the event structure can be utilized to describe the senses of verbs belonging to the three types above. From the operational definition, event structure can be represented by the event structure template which varies a lot, while we incline to adopt Levin \& Rappaport's descriptive methods.

Based on Vendler (1967)'s classification of the English verbs, Rappaport \& Levin (1998) categorized events into three types, the activity event, the achievement event and the accomplishment event, which can be depicted by the event structure templates below:

$[\mathrm{X}$ ACT $<$ MANNER $>]$

[BECOME $[\mathrm{x}<\mathrm{STATE} / \mathrm{PLACE}>]]$

[x CAUSE [BECOME $[\mathrm{y}<\mathrm{STATE} / \mathrm{PLACE}>]]]$

(the activity event)

(the achievement event)

(the accomplishment event)

[[x ACT $<$ MANNER $>$ CAUSE [BECOME $[y<$ STATE/PLACE $>]]] \quad$ (the accomplishment event)

The event structure template is mainly composed of three elements: 1) the primitive predicate, such as ACT, BECOME and CAUSE, which is owned by all the verbs, representing the same structural features, and should be written in capital letters; 2) the semantic constant (in the angle brackets), which stands for the verbs' idiosyncratic semantic parts or the semantic roots like STATE, PLACE, MANNER and so on; 3) the participant, which means the participant in the event, expressed by variables like $\mathrm{x}$ and $\mathrm{y}$ when there is no concrete context. 
Both the activity event and the achievement event are simple events, whereas the accomplishment event is a complex one, which is the integration of two simple events, which can be called "template augmentation". For instance, the event structure of "break" is:

[[x ACT $<$ MANNER $>$ CAUSE [BECOME [y $<$ BROKEN $>$ ]]]

This complex event is made up by two sub-events, the activity event ([x $\left.\mathrm{ACT}_{<\mathrm{MANNER}>}\right]$ ) and the achievement event ([BECOME $[\mathrm{y}<\mathrm{BROKEN}>]])$, and they are linked by CAUSE, indicating the cause relation between the two events. Therefore, we call this kind of complex event "causal event" as well.

\section{The Analysis of the Chinese Phrasal VR Constructions' Event Structures}

\subsection{The Causality of the Chinese Phrasal VR Constructions}

Lü Shuxiang (1942) noted the fact that VR constructions have the nature of causality at the earliest. He argued that similar to the "causative sentence" in classical Chinese, there is also a kind of syntax can express causation in written vernacular Chinese, such as “把河开深” (BA-river-open-deep, open up the waterway deep). Wang Li (1943) was the first to define the VR construction, as naming it "causative construction", that all the predicates and their complements which have the causative relationship can be termed as "causative constructions".

The four types of VR constructions, represented by “哭累”, “哭湿”, “洗累” and “推倒”, have a causative relation between $\mathrm{V}$ and R, i.e. they can be split into two clauses that have causative relationship in each. For instance, “张三推倒了椅子” (Zhang San pushed over the chair) can be interpreted as that the sub-event, “张 三推椅子” (Zhang San pushed the chair) results in the consistent sub-event, “椅子倒了” (The chair fell over). Therefore, all the four constructions are accomplishment events, and they can be described by Levin \& Rappaport's accomplishment event structure template.

\subsection{The Event Structures of the Chinese Phrasal VR Constructions}

The event structure can be understood as a bridge linking the verb's lexical meaning and the syntax, so analyzing the verb's lexical meaning has to depend on the sentence which it belongs to. In order to facilitate the interpretation, we will analyze the VR structures on the basis of sentences. Firstly, we picked up typical example sentences, each containing one of the four VR constructions, from the grammar books and language corpus; then we modified the example sentences in line with our research requirement, including change the personal pronouns in a uniform way, converting the non-agentive subject sentences into the agentive subject sentences and so on. Table 1 has listed some examples.

Table 1. Some examples of the Chinese phrasal VR constructions.

\begin{tabular}{|c|c|}
\hline Original Sentences & Rewriting Sentences \\
\hline $\begin{array}{l}\text { 他哭累了。 } \\
\text { (He-cried-tired, He was tired from crying) (From CCL }{ }^{1} \text { corpus) }\end{array}$ & $\begin{array}{l}\text { 张三哭累了。 } \\
\text { (Zhang San-cried-tired, Zhang San was tired from crying) }\end{array}$ \\
\hline $\begin{array}{l}\text { 张三哭湿了手绢。 } \\
\text { (Zhang San-cried-wet-the handkerchief, Zhang San cried the } \\
\text { handkerchief wet) (From CCL corpus) }\end{array}$ & $\begin{array}{l}\text { 张三哭湿了手绢。 } \\
\text { (Zhang San-cried-wet-the handkerchief, Zhang San cried the } \\
\text { handkerchief wet) }\end{array}$ \\
\hline $\begin{array}{l}\text { 妈妈洗衣服洗累了。 } \\
\text { (Mother-washed-the clothes-washed-tired, Mother was tired from } \\
\text { washing clothes) (Shi Chunhong, 2008: p. 96) }\end{array}$ & $\begin{array}{l}\text { 张三洗衣服洗累了。 } \\
\text { (Zhang San-washed-the clothes-washed-tired, Zhang San was } \\
\text { tired from washing clothes) }\end{array}$ \\
\hline $\begin{array}{l}\text { 有人推倒椅子, 有人站在椅子上。 } \\
\text { (Someone-pushed-over-the chair, someone-stood-on-the chair, } \\
\text { Someone pushed over the chair, and some one stood on the chair. } \\
\text { (From CCL corpus) }\end{array}$ & $\begin{array}{l}\text { 张三推倒了 }{ }^{2} \text { 椅子。 } \\
\text { (Zhang San-pushed-over-the chair, Zhang San pushed over the } \\
\text { chair) }\end{array}$ \\
\hline
\end{tabular}

${ }^{1}$ CCL: the abbreviation of "Center for Chinese Linguistics" of Peking University.

${ }^{2}$ Concerned about the grammar, we added "了” (indicating the action has finished) behind the VR construction. 
According to Levin \& Rappaport's event structure theory, the accomplishment event should be expressed as [[x ACT <MANNER>] CAUSE [BECOME $[y<$ STATE $>]]]^{3}$, which can be utilized to describe the type of “哭累” and “哭湿” constructions with facility.

(5) [[张三 $\left.{ }_{1} \mathrm{ACT}_{\text {<哭> }}\right]$ CAUSE [BECOME [张三 ${ }_{2}<$ 累>]]] ${ }^{4}$

[[Zhang $\left.\operatorname{San}_{1} \mathrm{ACT}_{<\text {cry }>}\right]$ CAUSE [BECOME [Zhang $\operatorname{San}_{2}<$ tired $\left.\left.>\right]\right]$

(6) [[张三 $\left.\mathrm{ACT}_{\text {<哭 }}\right]$ CAUSE [BECOME [手绢 <湿>]]]

[[Zhang San $\left.\mathrm{ACT}_{<\mathrm{cry}}\right]$ CAUSE [BECOME [handkerchief < wet>]]]

However, if the predicative verb in the VR construction is a two-valence verb, like “洗” and “推” in “张三洗 衣服洗累了” (Zhang San-washed-the clothes-washed-tired, Zhang San was tired from washing clothes) and “张 三推倒了椅子” (Zhang San pushed over the chair) respectively, they have directly related objects, “衣服” (clothes) and “椅子” (chair), on the deep semantic level. But these elements can not be reflected in the existing event structure templates, in that the existing expressions only have two places for participants, $x$ and $y^{5}$. In order to solve this problem, we need to add one more participant behind the ACT, i.e. [[x ACT $<$ MANNER> $(y)]$ CAUSE [BECOME $[\mathrm{z}<$ STATE $>]]]^{6}$.

(7) [[张三 ${ }_{1} \mathrm{ACT}_{<\text {芜> }}$ 衣服] CAUSE [BECOME [张三 ${ }_{2}<$ 累>]]]

[[Zhang San1 ACT <wash $>_{\text {clothes] CAUSE [BECOME [Zhang San2<tired }>\text { ]]] }}$

(8) [[张三 $\mathrm{ACT}_{<\text {推> }}$ 椅子 ${ }_{1}$ ] CAUSE [BECOME [椅子 ${ }_{2}<$ 倒> ] ] ]

[[Zhang San $\mathrm{ACT}_{<\text {push }>}$ chair ${ }_{1}$ ] CAUSE [BECOME [chair ${ }_{2}<($ fall $)$ over $>$ ]] $]$

Croft W, Cresswell M J. (1991) and Croft W. (1998) considered the complex event structure as a "causal chain" in which there are three kinds of participants. One is the initiator, i.e. the causer of the event, similar to Langacker R. W. (1987)'s trajector. Since we mainly focus on the agentive subject sentence, the initiator will always refer to the person, like “张三” in the sentence “张三砍这些排骨砍钝了两把刀” (Zhang San-choppedthese-ribs-chopped-blunt-two-knives, Zhang San chopped these ribs with two knives blunt). One is the target of activity, indicting the entity which is going through a certain action, and it is the directly related target of the action, like “这些排骨” (these ribs) in the sentence above. The third one is the locus of affect, i.e. the entity related to the end of the action, similar to Langacker R. W. (1987)'s landmark, like “两把刀” (two knives) in the example sentence aforementioned. (Quoted in Chang J., 2003)

In accordance with the analysis above, the participant before the ACT is the initiator in the event structure of Chinese VR construction, like “张三”. The nominal composition coming after the ACT is the target of activity. When the verb is of one-valence, there will be no target of activity, while, when the verb is of two-valence, there will be one target of activity. All the participants behind BECOME are locus of affect. When the verb is of onevalence, there will be one locus of affect.

When analyzing the event structures of Chinese VR constructions, we need to not only describe the event structures by event structure templates, but also consider the relationship among the participants. After observing 11 types of Chinese VR constructions, a good many participants have the co-reference relations, entailing that we have to consider which participant to be prominent and which should be recessed. Chang J. (2003) ordered the three kinds of participants in the light of importance: initiator $\rightarrow$ locus of affect $\rightarrow$ target of activity (from the most important to the least important). Thus, during the process of projection from the event structure to the syntactic structure, the initiator will be the easiest to be prominent, and the target of affect will be the easiest to be recessed.

From the perspective of the transmission of energy, the initiator is the source of energy, which should be the most prominent in a scene, and therefore it is always construed as the main focus by being put into the subject place of a sentence. The reason why the target of activity must be prominent in the verb-copying sentence is that it can explain the cause of the corresponding result, while the verb-copying sentence is used to provide the background information, i.e. (Zhang Wangxi, 2009: p. 59). So, the target of activity is not the focus. The locus of affect is the end of the energy transmission, whose resultant state should receive the cognitive highlighting, so it is the sub-focus in the whole scene, whose importance ranks only second to the initiator.

To sum up, for purpose of embodying the relations among the participants in the event structure template, we

\footnotetext{
${ }^{3}$ Since the Chinese VR construction is not correlated with displacement, we will not take the semantic constant, PLACE in, into account. ${ }^{4}$ If two participants in an expression are referred to the same entity, we will use the subscript 1 and 2, hereinafter the same.

${ }^{5}$ Rappaport Hovav M, Levin B. (1998) also mentioned the situation when there are three participants: [[x ACT $<$ MANNER> y] CAUSE. [BECOME [ $<$ PLACE $>]]$, and here, y denotes the implicit object of the verb which will not appear in the syntactic structures. For example in "Phil swept the crumbs onto the table", $\mathrm{x}$ is Phil, $\mathrm{y}$ is the surface, and $\mathrm{z}$ is the crumbs.

${ }^{6}$ The elements in the brackets are not necessary, hereinafter the same.
} 
will use brackets to denote the recession of certain participants and re-describe the four types of Chinese VR constructions as below:

(9) [[张三 $\left.{ }_{1} \mathrm{ACT}_{\text {<哭 }}\right]$ CAUSE [BECOME [(张三 $\left.{ }_{2}\right)<$ 累> $\left.\left.]\right]\right]$

[Zhang San1 $\left.\mathrm{ACT}_{<\mathrm{cry}}\right]$ CAUSE [BECOME [(Zhang $\left.\mathrm{San}_{2}\right)<$ tired $>$ ]]]

(10) [[张三 $\left.\mathrm{ACT}_{\text {<哭 }}\right]$ CAUSE [BECOME [手绢<湿>]]]

[[Zhang San $\left.\mathrm{ACT}_{<\mathrm{cry}}\right]$ CAUSE [BECOME [handkerchief $<$ wet $>$ ]]]

(11) [[张三 ${ }_{1} \mathrm{ACT}_{<\text {洗> }}$ 衣服] CAUSE [BECOME [(张三 $\left.{ }_{2}\right)<$ 累>]]]

[[Zhang San1 $\mathrm{ACT}_{<\text {泣> }}$ clothes] CAUSE [BECOME [(Zhang San2)<tired $>$ ]]]

(12) [[张三 $\mathrm{ACT}_{<\text {推> }}\left(\right.$ 椅子 $\left.\left.{ }_{1}\right)\right]$ CAUSE [BECOME [椅子 ${ }_{2}<$ 倒>] ] ]

[[Zhang San $\mathrm{ACT}_{<\text {push }>}\left(\right.$ (haii $\left._{1}\right)$ ] CAUSE [BECOME [chair ${ }_{2}<$ (full) over $>$ ]]]

According to the study above, we concluded some rules of Chinese phrasal VR constructions:

1. No matter under what circumstances, the initiator must be prominent;

2. All the targets of activity must be recessed so long as they are in co-reference.

3. Except from being co-referential to the initiator, all the locus of affect will be prominent.

\subsection{The Projection from Event Structure to Syntactic Structure}

After solving the problem of description of the event structure, it is time to study the process of the projection from event structure to syntactic structure. During the analysis, we will not take the participants in the brackets of the event structure template into account, in that they have been recessed, which have no possibility to be projected into the syntactic structure. What we do consider is which syntactic position the prominent participants will be projected to and in what way, direct projection or shift projection (means that the participant's original place will be changed when realized in the syntactic structure by projection).

We will first analyze the VR construction represented by “哭累”. The initiator “张三 1 ” should be directly projected into the subject of the syntactic structure, and the verb “哭” should be combined with the complement “累” as the VR construction “哭累” (See Figure 1).

In the VR constructions represented by “哭湿”, both the initiator “张三” and the locus of affect “手绢” are prominent. The initiator should be projected into the subject of the syntactic structure, after which the verb “哭” should be combined with the complement “湿” as the VR construction “哭湿”. The locus of affect should be projected into the object of the syntactic structure, but there is a transformation of the place of the locus of affect: at the deep semantic level, the locus of affect “手绢” is ahead of the complement “湿”, while in the syntactic structure, “手绢” is coming after “湿”. We will use a shadow to show the shift projection process (see Figure 2).

In the constructions represented by “洗累”, the initiator “张三 ${ }_{1}$ ” and the target of activity “衣服” are both prominent. The initiator should be projected into the subject of the syntactic structure, and after the subject, the verb “洗” should be integrated with the complement “累” so as to form the VR construction “洗累”. The locus of affect is not projected into the object of the VR construction, but urges the syntactic structure to copy the verb “洗”. And then it will be projected into the object of the verb, ahead of the VR construction (see Figure 3).

In the constructions represented by “推倒”, the initiator “张三” and the locus of affect “椅子 2 ” are prominent.

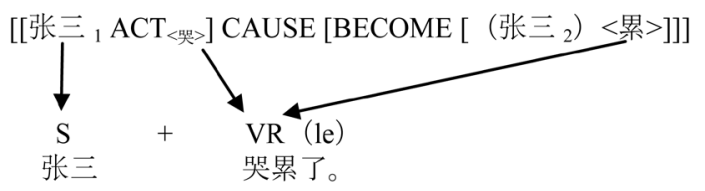

Figure 1. The projection process of “哭累”.

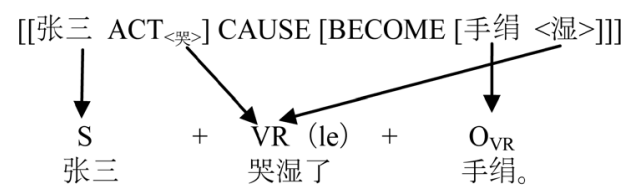

Figure 2. The projection process of “哭湿”. 


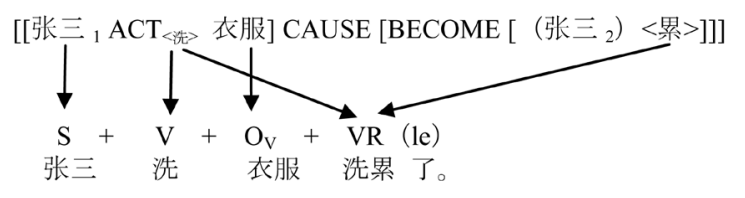

Figure 3. The projection process of “洗累”.

The initiator should be projected to the subject position of the syntactic structure, and after the subject, the verb “推” should be combined with the complement “倒” so as to form the VR construction “推倒”. The locus of affect is projected to the object position of the VR construction with the transformation of its place, i.e. at the deep semantic level, the locus of affect “椅子” is in front of the complement “倒”, while in syntactic structure, “椅子” falls behind “倒” (see Figure 4).

Similarly, we concluded the principles of the projection from event structure of Chinese phrasal VR constructions to syntactic structure:

1. The initiator must be projected to the subject position of the syntactic structure.

2. The locus of affect must be projected to the object position of the VR construction. If there is a locus of affect in the event structure, there will be a shifting projection (with the transformation of its place), i.e. at the deep semantic level, the locus of affect is before the complement, while in the syntactic structure, it will come after the complement.

3. The target of activity requires the syntactic structure to copy the verb and then it will be projected to the object position of this verb, ahead of the VR construction.

4. During the integration of the verb and complement as the VR construction, if the target of activity is prominent, it will entail the syntactic structure to copy the verb, and then be projected to the object position of the verb, lying before the VR construction.

We tried to find out the cognitive proofs of the principles above so as to interpret them. According to Tai's "the principle of temporal sequence", the two syntactic units' order is defined by their temporal sequence in the cognitive domain. Whereas, the cognitive domain is the representation of the objective events in brain. Therefore, the comparative order of the two syntactic units is decided by the occurrence order of the objective events. In light of this view, in the event structures of the Chinese VR constructions, the activity event should happen before the achievement event. Thus, the participant of the activity event should be projected to those syntactic positions before the participant of the achievement structure. Still taking “张三砍排骨砍钝了两把刀” for instance, “张三砍排骨” is an activity event, and “两把刀钝了” is an achievement event. When projected to the syntactic structure, the order of the three participants should be "the initiator-the target of activity-the locus of affect". The initiator must be projected to the subject position, but how to arrange the positions of the target of activity and the locus of affect? There will be three possibilities ${ }^{7}: 1$ ) both the target of activity and the locus of affect can be projected to object positions of VR, with the former one being the object closed to the VR construction and the latter one the object far away from the VR construction, i.e. S + VR $+\mathrm{O}_{\mathrm{VR}}^{1}$ (the target of activity) $+\mathrm{O}_{\mathrm{VR}}^{2}$ (the locus of affect); 2) by copying the verb, both the target of activity and the locus of affect can be projected to the object positions of the verb, with the former one being the object closed to the verb and the latter one being the object far away from the verb, i.e. $\mathrm{S}+\mathrm{V}+\mathrm{O}_{\mathrm{v}}^{1}$ (the target of activity) $+\mathrm{O}_{\mathrm{v}}^{2}$ (the locus of affect) + VR; 3) by copying the verb, the target of activity should be projected to the object position of the verb, and the locus of affect would be projected to the object position of the VR construction, i.e. $\mathrm{S}+\mathrm{V}+\mathrm{O}_{\mathrm{V}}$ (the target of activity) $+\mathrm{VR}+\mathrm{O}_{\mathrm{VR}}$ (the locus of affect).

At the deep semantic level, the target of activity is directly related to the action, whose meaning is comparatively closed to the verb, and the complement reflecting the result state is therefore more closed to the locus of affect. According to the "distance iconicity principle", the distance of concept decides the syntactic distance, so that when projected to the syntactic position, the target of activity's position ought to be more closed to the verb, while the locus of affect should go close to the position of the complement. As to the Chinese VR constructions, the closest position to the complement is the object position of VR construction. Therefore, we may deduce that the locus of affect should be projected to the object position of VR construction with a positional transformation, i.e. originally the locus of affect is in front of the complement, but after projection, it goes behind the complement (VR construction). And the position closest to the verb should be the object position of the verb. However,

${ }^{7}$ Here, we will put the situation that two targets of activity or two locuses of affect are prominent aside. 


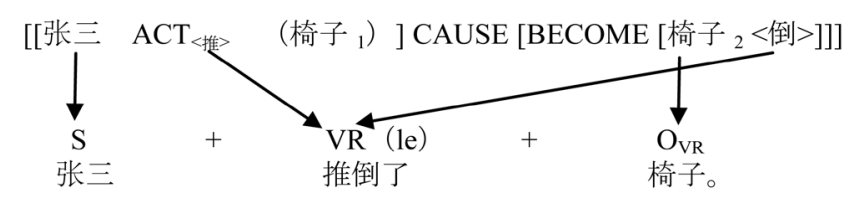

Figure 4. The projection process of “推倒”.

the object and the complement can not co-exist behind the verb, so in order to solve this contradiction known as "the competition for the verb of object and complement", the syntactic structure has to copy the verb to create a new position before the VR construction, which is also the motive of the creation of the verb-copying sentences (Pei Xiaoyan, 2007; Sun Hongling, 2005). Therefore, when the target of activity is prominent, the verb needs to be copied, and then the target of activity will be projected into the object of the verb. In summary, if both the target of activity and the locus of affect are prominent, then after projection, the syntactic structure should be expressed as " $\mathrm{S}+\mathrm{V}+\mathrm{O}_{\mathrm{V}}$ (the target of activity) $+\mathrm{VR}+\mathrm{O}_{\mathrm{VR}}$ (the locus of affect)".

\section{Conclusion}

Now we may answer the questions arising in the introduction part, and we will use “张三” to substitute all the subjects of the example sentences.

Question 1: Both “哭湿” and “哭累” are combined by the one-valence verb (which can only have one argument) “哭” and the one-valence adjective “湿” or “累”, but why can only the “哭湿” have the object?

There is no prominent locus of affect in the event structure of “哭累”, so the locus of affect can not be projected to the object position of VR construction. But “手绢”, the locus of affect in the event structure of “哭湿” is prominent. So it can be realized as the object of the VR construction. See examples below:

(16) 张三哭累了。

Zhang San-cried-tired.

Zhang San was tired from crying.

*张三哭累了张三。

*Zhang San-cried-tired-Zhang San.

(17) *张三哭湿了。

*Zhang San-cried-wet.

张三哭湿了手绢。

Zhang San-cried-wet-the handkerchief.

Zhang San cried the handkerchief wet.

Question 2: Both “洗累” and “推倒” are made up by the two-valence verb “洗” or “推” and the one-valence adjective “累” or “倒”; however, why can not “洗累” behave the same as “推倒”, taking an object directly, but have to repeat the main verb “洗” to bring the object forward?

“椅子” is the locus of affect, which therefore can be projected to the object position of VR construction, while “衣服” is the target of activity, so that it can only be projected to the object position of the repeated (or copied) verb. See examples below:

(18) 张三推倒了椅子。

Zhang San-pushed-over-the chair.

Zhang San pushed over the chair.

*张三推椅子推倒了。

*Zhang San-pushed-the-chair-pushed-over.

(19) *张三洗累了衣服。

*Zhang San-washed-tired-the clothes.

张三洗衣服洗累了。

Zhang San-washed-the clothes-washed-tired.

Zhang San was tired from washing the clothes.

In conclusion, having finished the description of both the event structures of the four types of Chinese VR constructions and the projection process from the event structures to the syntactic structures, we not only explained the questions remained unsolved in the studies before, but also provided the future second language acquisition researches with a foundation, especially hoping our result would contribute both theoretical and prac- 
tical references to the field of teaching Chinese as a second language.

\section{Acknowledgements}

This article has received supports from the key project of the National Social Science Fund (NO. 12AZD113) and the general project of the National Social Science Fund (NO. 14BYY146).

\section{References}

Chang, J. (2003). Event Structure and Argument Linking in Chinese. Language and Linguistics, 4, 317-351.

Croft, W. (1998) Event Structure in Argument Linking. The Projection of Arguments: Lexical and Compositional Factors, 8 , 21-63.

Croft, W., \& Cresswell, M. J. (1991). Syntactic Categories and Grammatical Relations: The Cognitive Organization of Information. Chicago and London.

Langacker, R. W. (1987). Foundations of Cognitive Grammar: Theoretical Prerequisites (Vol. 1). Redwood City, CA: Stanford University Press.

Lü, S. X. (1942). Essentials of Chinese Grammar. Beijing: The Commercial Press.

Pei, X. Y. (2007). The Research of V-R in Verb-Copying Sentences. Master Dissertation, Shanghai: Shanghai Normal University.

Rappaport Hovav, M., \& Levin, B. (1998). Building Verb Meanings. The Projection of Arguments: Lexical and Compositional Factors, 97-134.

Shi, C. H. (2008). The Syntactic and Semantic Studies of Chinese V-R. Beijing: Beijing Language University Press.

Sun, H. L. (2005). The Study of Verb-copying Sentences in Modern Chinese. PhD Dissertation, Beijing: Beijing Language University.

Vendler, Z. (1967). Linguistics in Philosophy. Ithaca: Cornell University Press.

Wang, L. (1943). Modern Chinese Grammar. Bejing: The Commercial Press.

Zhang, W. X. (2009). Studies on Covert Quantity of Syntactic Structures in Chinese. Beijing: Beijing Language University Press. 
Scientific Research Publishing (SCIRP) is one of the largest Open Access journal publishers. It is currently publishing more than 200 open access, online, peer-reviewed journals covering a wide range of academic disciplines. SCIRP serves the worldwide academic communities and contributes to the progress and application of science with its publication.

Other selected journals from SCIRP are listed as below. Submit your manuscript to us via either submit@scirp.org or Online Submission Portal.
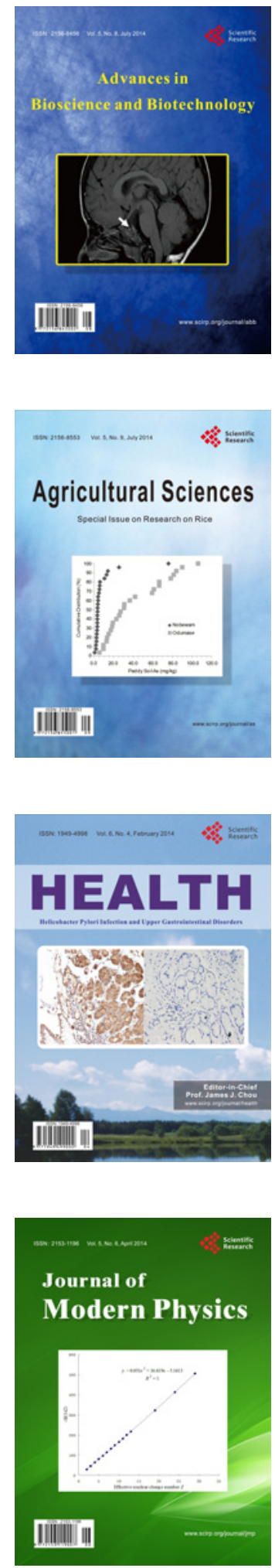
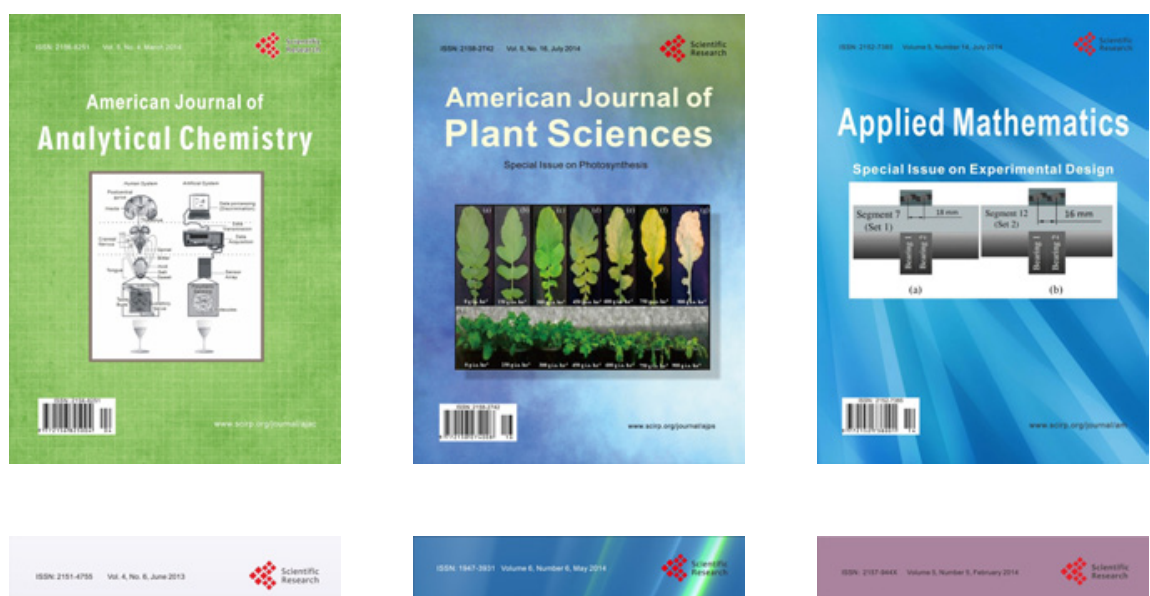

Creative Education
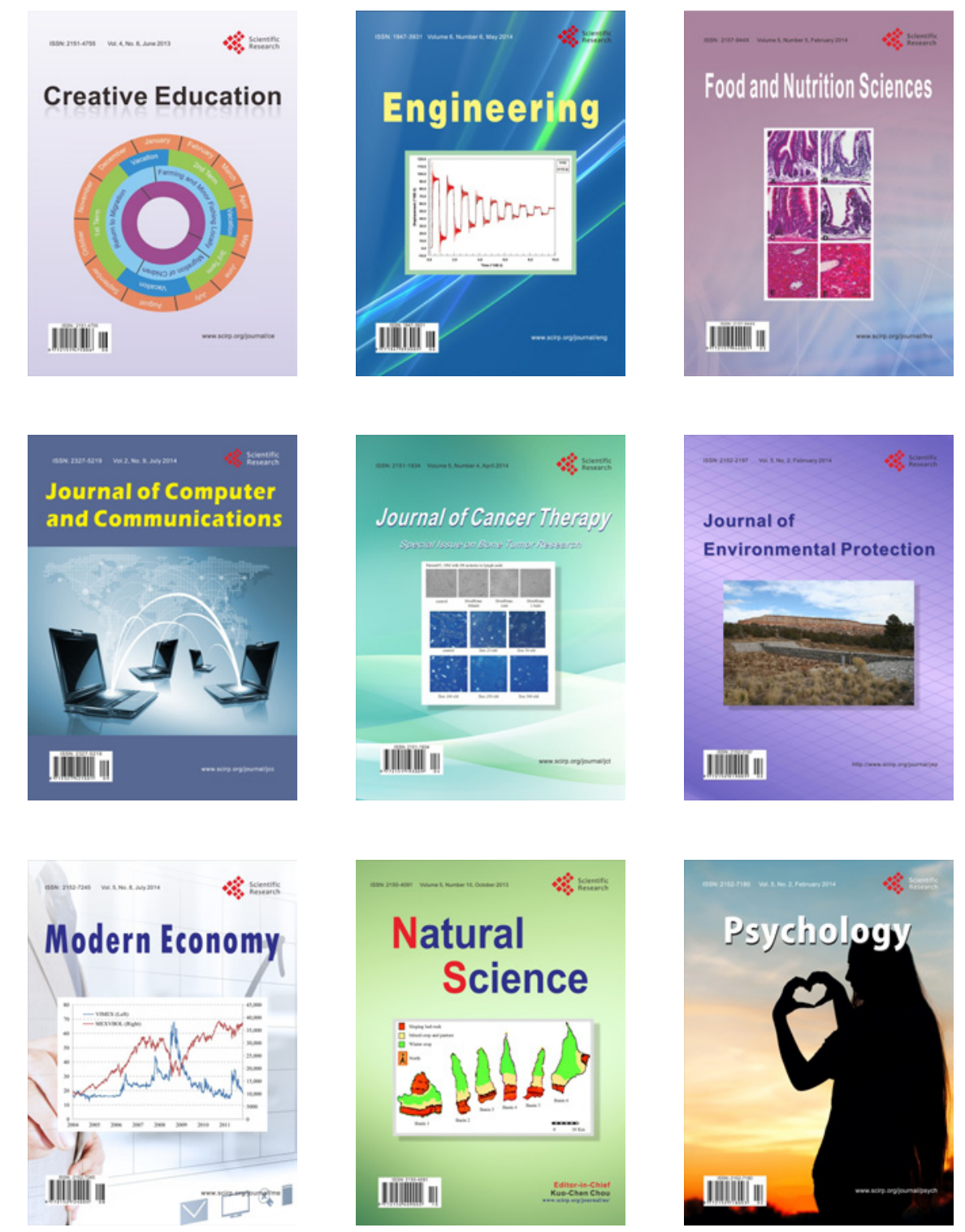